density remains on the nickel. This seems to be an unreasonably small amount, especially since the $\mathrm{Ni}^{3+}$ superhyperfine structure indicates only about $18 \%$ density on the neighboring ligands. The greater the positive charge of an ion, the greater should be its participation in covalent bonding with the neighboring anions.

The $\mathrm{Ni}^{+} \mathrm{g}$ factor may be reduced also by the JahnTeller effect. The theory in this case would be complicated. The nickel $t_{2}$ functions can interact with two $t_{2}$ normal modes of the tetrahedral cluster and also with the $e$ mode. The spin-orbit coupling constant of $\mathrm{Ni}^{+}$ is rather large and may be comparable with the JahnTeller interaction, so that the usual approximations ${ }^{16}$ made in treating such a problem would not be valid.

\section{ACKNOWLEDGMENTS}

I thank W. C. Holton and M. de Wit for helpful comments, and R. D. Stinedurf for preparing the $\mathrm{ZnSe}: \mathrm{Ni}$ crystals.

${ }^{16}$ M. D. Sturge, in Solid State Physics, edited by F. Seitz, D. Turnbull, and $\mathrm{H}$. Ehrenreich (Academic Press Inc., New York, 1967), Vol. 20, p. 92 (and references therein).

\title{
Influence of Simultaneous Static and Time-Dependent Quadrupole Interactions on Gamma-Gamma Angular Correlations*
}

\author{
P. da R. Andrade, J. D. Rogers, and A. Vasque\% \\ Instituto de Fisica, liniversidade Federal do Rio Girande do Sul, Pôrto Alesre, Brasil
}

(Received 11 July 1969)

\begin{abstract}
We derive the form of the perturbation factors for the angular correlation of two successive $\gamma$ rays when the intermediate state is under the influence of an axially symmetric static electric field gradient plus a small randomly fluctuating quadrupole perturbation. The Bloch-Wangness-Redfield theory of nuclear relaxation is applied to calculate the evolution of the density matrix, and the form of the perturbation factors is calculated for several special cases in single-crystal and powder sources.
\end{abstract}

\section{INTRODUCTION}

$\mathrm{D}$ URING the past several years the study of nuclear hyperfine interactions by perturbed angular correlation techniques has been shown to be a profitable way of obtaining new information about solid- and liquid-state problems. The great variety of experimental information that can be obtained is illustrated in Refs. 1 and 2, and as experimental technique develops it is to be expected that the field will become even broader. One area of particular interest that can be studied by these techniques seems to be the detection of nuclear relaxation processes in solids.

Relaxation effects on angular correlations have previously been discussed for special cases by Abragam and Pound, ${ }^{3}$ who treated the case of isotropic fluctuations in liquids, by $\mathrm{Micha}^{4}$ for the case of magnetic relaxation in solids, and by Tang and Osborn, ${ }^{5}$ who discussed relaxation due to crystalline vibrations.

* Work partially supported by Conselho Nacional de Pesquisas (Brasil), Conselho de Psequisas (UFRGS), and U. S. Air Force Office of Scientific Research, Grant No. AF-AFOSR-1280-67.

1 Perturbed Angular Correlations, edited by E. Karlsson, E. Matthias, and K. Sieghbahn (North-Holland Publishing Co., Amsterdam, 1964).

${ }^{2}$ Hyperfine Struclure and Nuclear Radiations, edited by E. Matthias and D. A. Shirley (North-Holland Publishing Co. Amsterdam, 1968).

${ }^{3}$ A. Abragam and R. V. Pound, Phys. Rev. 92, 953 (1953).

${ }^{4}$ D. A. Micha, Phys. Rev. 156, 627 (1967).

${ }^{5}$ L. H. Tang and R. K. Osborn, Phys. Rev. 146, 695 (1966).
In this paper we present an application of the BlochWangness-Redfield theory of nuclear relaxation to the calculation of the perturbation factors for angular correlations of successive $\gamma$ radiations in solids. In particular, we treat the case of an odd- $A$ nucleus in the presence of an axially symmetric electric field gradient and a weak fluctuating perturbation; emphasis is given to quadrupole relaxation effects as an example demonstrating the effects to be expected from some typical relaxation mechanisms in solids, such as molecular torsion oscillations and planar and isotropic hindered rotations. ${ }^{6}$ The extension to more general cases is discussed briefly.

In Sec. II, we develop the density matrix describing the intermediate state as a result of the combined static and fluctuating perturbations; in Sec. III, we show the application of the resultant density matrix elements to the calculation of the perturbation factor for several different experimental situations, and in Sec. IV a discussion of the results is presented.

\section{EVOLUTION_OF DENSITY MATRIX}

The angular correlation function of two successive $\gamma$ radiations emitted in directions specified by the wave vectors $\mathbf{k}_{\mathbf{1}}$ and $\mathbf{k}_{2}$, respectively, and separated by a time

${ }^{6}$ A. Abragam, The Principles of Nuclear Magnetism (Oxford University Press, London, 1961), Chaj!. X. 
interval $t$ during which a perturbation acts on the intermediate state can be written as

$$
\begin{aligned}
W\left(\mathbf{k}_{\mathbf{1}}, \mathbf{k}_{2}, t\right) & =\operatorname{Tr}\left\{\rho\left(\mathbf{k}_{\mathbf{1}}, t\right) \rho\left(\mathbf{k}_{2}, 0\right)\right\} \\
& =\sum_{\alpha, \alpha^{\prime}}\left\langle\alpha\left|\rho\left(\mathbf{k}_{1}, t\right)\right| \alpha^{\prime}\right\rangle\left\langle\alpha^{\prime}\left|\rho\left(\mathbf{k}_{2}, 0\right)\right| \alpha\right\rangle,
\end{aligned}
$$

where $\rho\left(\mathbf{k}_{2}, 0\right)$ is the density matrix of the second radiation and has matrix elements

$$
\begin{aligned}
& \left\langle\alpha^{\prime}\left|\rho\left(\mathbf{k}_{2}, 0\right)\right| \alpha\right\rangle \\
& \quad=(4 \pi)^{1 / 2} \sum_{k_{1}}(-1)^{\alpha} A_{k_{2}}\left(\begin{array}{ccc}
I & I & k_{2} \\
\alpha^{\prime} & -\alpha & \mu^{\prime}
\end{array}\right) Y_{k_{2} \mu^{\prime}}\left(\mathbf{k}_{2}\right)
\end{aligned}
$$

and $\rho\left(\mathbf{k}_{\mathbf{1}}, 0\right)$ is the density matrix describing the first radiation with matrix elements

$$
\begin{aligned}
& \left\langle\alpha\left|\rho\left(\mathbf{k}_{1}, 0\right)\right| \alpha^{\prime}\right\rangle \\
& \quad=(4 \pi)^{1 / 2} \sum_{k 2}(-1)^{\alpha} A_{k_{1}}\left(\begin{array}{ccc}
I & I & k_{1} \\
\alpha^{\prime} & -\alpha & \mu
\end{array}\right) Y_{k_{1} \mu} *\left(\mathbf{k}_{1}\right) .
\end{aligned}
$$

The factors $A_{k_{1}}$ and $A_{k_{2}}$ appearing in Eqs. (2.1) and (2.2) are the usual angular correlation coefficients. ${ }^{7}$

During the time the nucleus is in the intermediate state, interactions of extra nuclear fields with the electric quadrupole and magnetic dipole moments of the nucleus will cause changes in the density matrix of the intermediate state. If $H$ is the Hamiltonian describing this interaction, then the evolution of the density matrix is governed by the differential equation

$$
\dot{\rho}=(-i / \hbar)[H, \rho]
$$

with the boundary condition given by Eq. (2.3) above.

We are interested in the solution of Eq. (2.4) for the special case where $H$ is composed of a strong static field $K_{0}$ and a weak randomly fluctuating time-dependent interaction $K(t)$. We follow the development given in Refs. 6, 8, and 9. If

we have

$$
H=K_{0}+K(t),
$$

which has the immediate formal solution

$$
\rho(t)=e^{-(i / \hbar) K_{0} t} \rho^{*}(t) e^{(i / \hbar) K_{0} t},
$$

where $\rho^{*}(t)$ is the solution of the differential equation

$$
\dot{\rho}^{*}=(-i / \hbar)\left[K^{*}(t), \rho^{*}\right] \text {. }
$$

As is shown in Refs. 6, 8, and 9, Eq. (2.8) can be written under certain conditions in the form

$$
\dot{\rho}^{*}=-\int_{0}^{\infty}\left[K^{*}(t),\left[K^{*}(t-\tau), \rho^{*}(t)\right]\right] d t .
$$

${ }^{7}$ K. Alder and R. M. Steffen, Ann. Rev. Nucl. Sci. 14, 403 (1964).

${ }^{8} \mathrm{C}$. P. Slichter, Principles of Magnetic Resonance (Harper and Row Publishers, Inc., New York, 1963), Chap. 5.

${ }^{9}$ A. G. Redfield, IBM J. Res. Develop. 1, 19 (1957).
The most important of these conditions for our purposes are:

(a) There exists a time $\tau_{c}$ such that for $\tau \gg \boldsymbol{\tau}_{c}$ the correlation function defined as

$$
g(\tau)=\frac{1}{2 T} \int_{-T}^{T} K(t) K(t-\tau) d t
$$

is zero. $\tau_{c}$ is called the correlation time.

(b) The perturbation $K(t)$ is "small enough." This is specified by the requirement that there exist times $t$ such that

$$
\left|K^{2}\right| \tau_{c} t \ll 1 \text { and } t \gg \tau_{c}
$$

simultaneously.

(c) The function $K(t)$ is a stationary random function, that is, the average of $K(t)$ over time is equal to the average over ensembles.

(d) It is assumed that the time average of the fluctuating field is zero. However, if this is not the case the requirement can be satisfied by a suitable redefinition of the static perturbation $K_{0}$.

(e) Finally the solution of Eq. (2.9) above applies only for times $t$ larger than $\tau_{c}$.

Equation (2.9) can be written in a matrix form as

$$
\dot{\rho}_{\alpha \alpha^{\prime}} *=\sum_{\beta \beta^{\prime}} R_{\alpha \alpha^{\prime} \beta \beta^{\prime}} e^{i\left(\alpha-\alpha^{\prime}-\beta+\beta^{\prime}\right) t} \rho_{\beta \beta^{\prime}} *(t) .
$$

In this expression the subscripts $\alpha$ and $\beta$ refer to eigenstates of the Hamiltonian $K_{0}$, while the symbols $\alpha$ and $\beta$ appearing within the parentheses are abbreviated notations for the angular frequencies $\omega_{\alpha}=E_{\alpha} / \hbar$ of those states. This standard notation will be used throughout.

The definition of the matrix $R_{\alpha \alpha^{\prime} \beta \beta^{\prime}}$ in terms of the matrix elements of the Hamiltonian $K(t)$ is given by

$$
\begin{aligned}
R_{\alpha \alpha^{\prime} \beta \beta^{\prime}} & =\frac{1}{2 \hbar^{2}}\left[\mathcal{J}_{\alpha \beta \alpha^{\prime} \beta^{\prime}}\left(\alpha^{\prime}-\beta^{\prime}\right)+\mathcal{J}_{\alpha \beta \alpha^{\prime} \beta^{\prime}}(\alpha-\beta)\right. \\
-\delta_{\alpha^{\prime} \beta^{\prime}} & \left.\sum_{\gamma} \mathcal{J}_{\gamma \beta \gamma \alpha}(\gamma-\beta)-\delta_{\alpha \beta} \sum_{\gamma} \mathcal{J}_{\gamma \alpha^{\prime} \gamma \beta^{\prime}}\left(\gamma-\beta^{\prime}\right)\right],
\end{aligned}
$$

where the spectral densities $\mathscr{J}(\omega)$ are given by

$$
\begin{array}{r}
\oint_{\alpha \alpha^{\prime} \beta \beta^{\prime}}(\omega)=\int_{-\infty}^{\infty}\left\langle\left(\alpha|K(t)| \alpha^{\prime}\right)\left(\beta^{\prime}|K(t-\tau)| \beta\right)\right\rangle_{e} \\
\times e^{-i \omega \tau} d \tau,
\end{array}
$$

where \langle\rangle$_{e}$ means an ensemble average. We restrict ourselves to Hamiltonians of the form

$$
K(t)=\sum_{\mu} f_{\mu}^{q}(t)\left[K_{\mu}^{q}+\left(K_{\mu}^{q}\right)^{*}\right],
$$

where the $K_{\mu}{ }^{q}$ are tensor operators of rank $q$, and the $f_{\mu}{ }^{q}(t)$ are real random functions of time. We will be especially interested in the case $q=2$ (quadrupole perturbations). 
We further specialize to the case where the static Hamiltonian $K_{0}$ is axially symmetric, which allows the labels $\alpha$ and $\beta$ to be taken as magnetic quantum numbers. If this is the case, the spectral density functions can be written using the Wigner-Eckart theorem as

$$
\begin{array}{r}
g_{\alpha \alpha^{\prime} \beta \beta^{\prime}}=\int_{-\infty}^{\infty} \sum_{\mu} g_{\mu}{ }^{q}(\omega)\left\langle I \alpha^{\prime} q \mu \mid I \alpha\right\rangle\left\langle I \beta q \mu \mid I \beta^{\prime}\right\rangle \\
\times \frac{\left|\left\langle I\left\|K^{q}\right\| I\right\rangle\right|^{2}}{2 I+1},
\end{array}
$$

where the $g_{\mu}{ }^{q}(\omega)$ are given by

$$
g_{\mu}{ }^{q}(\omega)=\int_{-\infty}^{\infty} f_{\mu}^{q}(t) f_{\mu}^{q}(t-\tau) e^{i \omega \tau} d \tau
$$

and $f_{\mu}(t)$ and $f_{\mu^{\prime}}(t)$ are assumed to be uncorrelated.

In solving Eq. (2.10) above, it is usual to restrict oneself to those matrix elements for which $E_{\alpha}-E_{\alpha^{\prime}}$ $-E_{\beta}+E_{\beta^{\prime}}=0$, that is, to those terms for which $e^{i\left(\alpha-\alpha^{\prime}-\beta+\beta^{\prime}\right) t}=1$. (The reasoning behind this approximation and its limitations are discussed in the references, especially in Ref. 3.) The relaxation equation (2.10) then becomes

$$
\dot{\rho}_{\alpha \alpha^{\prime}}{ }^{*}=\sum_{\beta \beta^{\prime}} R_{\alpha \alpha^{\prime} \beta \beta^{\prime}} \rho_{\beta \beta^{\prime}}{ }^{*}, \quad\left(\alpha-\alpha^{\prime}\right)=\left(\beta-\beta^{\prime}\right) .
$$

These equations have the form of an eigenvalue problem and, for given values of the matrix elements $R$, can be solved using the standard techniques for such problems.

If we assign an index $\nu$ to each pair of levels $\alpha \alpha^{\prime}$, the element $\rho_{\nu}{ }^{*}(t)$ of the density matrix can be written as

$$
\rho_{\nu}^{*}(t)=\sum_{r} b_{r \nu} e^{-\lambda_{r} t} \rho_{\nu}^{* r}(0)
$$

where the $\rho_{\nu}{ }^{* r}(0)$ are the eigensolutions of Eq. (2.16) and have the form

$$
\rho_{\nu}^{* r}(0)=\sum_{\mu} c_{r \mu} \rho_{\mu \nu}{ }^{*}(0)
$$

and furthermore the matrix $c_{r \mu}$ is the inverse of the matrix $b_{r \nu}$.

In the general case it is necessary to solve Eq. (2.16) numerically to obtain values for the eigenvalues and the expansion coefficients $c_{r \mu}$.

Substituting Eq. (2.18) into Eq. (2.17), we obtain the usual form

$$
\rho_{\nu}^{*}(t)=\sum_{\mu}\left\langle\nu\left|G^{*}(t)\right| \mu\right\rangle \rho_{\mu}^{*}(0)
$$

where

$$
\left\langle\nu\left|G^{*}(t)\right| \mu\right\rangle=\sum_{r} b_{r \nu} c_{r \mu} e^{-\lambda_{r} t}
$$

The physical case we treat here is that of an odd- $A$ nucleus in an axially symmetric electric field gradient. In this case, the levels are pairwise degenerate with
$E_{\alpha}=E_{-\alpha}$, where $\alpha$ is the projection of the nuclear spin on the symmetry axis of the field gradient (Kramers doublets). Further, no two energy separations between different pairs of levels are equal.

In this case, it can be seen by inspection that an important simplification can be made in the set of equations represented by Eq. (2.16). Namely, it can be seen that there is no coupling between elements of the type $\rho_{\alpha \alpha}{ }^{*}$ and those of the type $\rho_{\alpha \alpha^{\prime}}{ }^{*}$. The "diagonal" elements are uncoupled from the "off-diagonal" elements. Thus these two cases can be treated separately.

In the particular case of the off-diagonal elements, the fact that no two pairs of levels have the same energy separation means that in the equation for the density matrix element $\rho_{\alpha \alpha^{\prime}} *(t)$ only four terms can contribute in the right-hand side. These are $R_{\alpha \alpha^{\prime} \beta \beta^{\prime}}, R_{\alpha \beta-\alpha-\beta}$, $R_{\alpha \alpha^{\prime}-\beta-\beta^{\prime}}$, and $R_{\alpha \alpha^{\prime} \beta-\beta^{\prime}}$. With a Hamiltonian of the form of Eq. (2.13), only the first of these turns out to be nonzero. Thus Eq. (2.16) for the off-diagonal elements has the simple form

$$
\dot{\rho}_{\alpha \alpha^{\prime}}{ }^{*}=R_{\alpha \alpha^{\prime} \beta \beta^{\prime}} \rho_{\beta \beta^{\prime}}{ }^{*},
$$

and the solutions are

with

$$
\begin{gathered}
\rho_{\alpha \alpha^{\prime}} *(t)=e^{-\lambda_{\alpha \alpha^{\prime} t} \rho_{\beta \beta^{\prime}} *(0),} \\
\lambda_{\alpha \alpha^{\prime}}=-R_{\alpha \alpha^{\prime} \beta \beta^{\prime}} .
\end{gathered}
$$

The equations for the diagonal elements do not have such a simple structure. They can be rearranged and written in the form

$$
\dot{\rho}_{\alpha \alpha}{ }^{*}=\sum_{\beta} \mathscr{J}_{\alpha \beta \alpha \beta}\left(\rho_{\beta \beta}{ }^{*}-\rho_{\alpha \alpha}{ }^{*}\right),
$$

with the formal solution [see Eq. (2.19)]

$$
\rho_{\alpha \alpha}{ }^{*}(t)=\sum_{\beta}\left\langle\alpha \alpha\left|G^{*}(t)\right| \beta \beta\right\rangle_{\rho_{\beta \beta}}{ }^{*}(0)
$$

In the general case, it would be necessary to calculate the values of the spectral density function using the Hamiltonian (2.13) and to solve Eq. (2.12) numerically, obtaining solutions of the form of (2.14) for each element. For the usual case where polarizations are not measured, a total of $\frac{1}{2}(2 I+1)$ relaxation constants would appear in various combinations for each element $\rho_{\alpha \alpha}$ of the density matrix at time $t$.

An important special case exists when the relaxation mechanism is isotropic, that is, when $g_{\mu}^{q}(\omega)$ in Eq. (2.15) is the same for all values of $\mu$ and $\omega$. It has been shown previously that in this case the relaxation constants are given by

$$
\lambda_{r}=\frac{1}{\hbar^{2}}\left[\sum_{\beta} g_{\alpha \beta \alpha \beta}-a_{r}\right],
$$

where $a_{r}$ is an eigenvalue of Eq. (2.23), and that the expansions coefficients $c_{r \mu}$ are proportional to vector coupling coefficients. 
The general case of isotropic perturbations has been considered in Ref. 10, where the necessary conditions for the expansion coefficients to be proportional to vector coupling coefficients are discussed. The angular correlation function has a particularly simple form when this is the case [see Eq. (3.3) below].

Starting from Eqs. (2.7) and (2.16), we can write the general form

$$
\rho_{\alpha \alpha^{\prime}}\left(\mathbf{k}_{1}, t\right)=\sum_{\beta \beta^{\prime}}\left\langle\alpha \alpha^{\prime}|G(t)| \beta \beta^{\prime}\right\rangle \rho_{\beta \beta^{\prime}}(0),
$$

where, from Eqs. (2.7) and (2.19), we see that

$$
\left\langle\alpha \alpha^{\prime}|G(t)| \beta \beta^{\prime}\right\rangle=e^{-i\left(\alpha-\alpha^{\prime}\right) t}\left\langle\alpha \alpha^{\prime}\left|G^{*}(t)\right| \beta \beta^{\prime}\right\rangle .
$$

Substituting, finally, Eq. (2.25) into Eq. (2.1), we can write the correlation function as

$$
\begin{aligned}
W\left(\mathbf{k}_{1}, \mathbf{k}_{2}, t\right)=4 \pi \sum_{k_{1} \mu} \sum_{k_{2} \mu^{\prime}} A_{k_{1}} A_{k_{2}} G_{k_{1} k_{2}}{ }^{\mu \mu^{\prime}}(t) \\
\\
\quad \times Y_{k_{1}{ }^{\mu *}}{ }^{*}\left(\mathbf{k}_{1}\right) Y_{k_{2}}{ }^{{ }^{\prime}}\left(\mathbf{k}_{2}\right),
\end{aligned}
$$

where we define the perturbation factors $G_{k_{1} k_{2}}{ }^{\mu \mu^{\prime}}(t)$ as

$$
\begin{aligned}
& G_{k_{1} k_{2}{ }^{\mu \mu^{\prime}}}(t)=\sum_{\alpha}\left(\begin{array}{ccc}
I & I & k_{1} \\
\alpha^{\prime} & -\alpha & \mu
\end{array}\right)\left(\begin{array}{ccc}
I & I & k_{2} \\
\alpha & -\alpha & \mu^{\prime}
\end{array}\right) e^{-i\left(\alpha-\alpha^{\prime}\right) t} \\
& \times\left\langle\alpha \alpha^{\prime}\left|G^{*}(t)\right| \beta \beta^{\prime}\right\rangle,
\end{aligned}
$$

and where the factors $\left\langle\alpha \alpha^{\prime}|G(t)| \beta \beta^{\prime}\right\rangle$ are to be calculated, as outlined in this section, from the eigensolutions of the diagonal and off-diagonal parts of the relaxation matrix. Equation (2.28) may be considered as the general form for the perturbation factors for sources in the form of single crystals.

\section{PERTURBATION FACTORS}

The above solutions (for the elements of the density matrix as a function of time) can now be used to derive the detailed form of the angular correlation function resulting from the combination of a static and a fluctuating perturbation.

The more usual experimental situation is that of a polycrystalline source. The perturbation coefficients for this case can be easily obtained using the technique of Ref. 11, which consists of averaging over the angles specified by $\mathbf{k}_{\mathbf{1}}$ and $\mathbf{k}_{\mathbf{2}}$ in Eq. (2.27) above while holding the angle between $\mathbf{k}_{\mathbf{1}}$ and $\mathbf{k}_{\mathbf{2}}$ constant. One obtains the general relation (valid for time-dependent, time-independent, and asymmetric perturbations)

$$
\bar{W}\left(\mathbf{k}_{\mathbf{1}}, \mathbf{k}_{2}, t\right)=\sum_{k} A_{k}^{(1)} A_{k}^{(2)} G_{k}(t) P_{k}(\cos \theta),
$$

where $\theta$ is the angle between $\mathbf{k}_{\mathbf{1}}$ and $\mathbf{k}_{\mathbf{2}}$ and where the

\footnotetext{
${ }^{10}$ D. Dillenburg and Th. A. J. Maris, Phys. Letters 17, 293 (1965).

${ }^{11}$ S. Devons and L. J. B. Goldfarb, in Handbuch der Physik, edited by S. Flügge (Springer-Verlag, Berlin, 1957), Vol. 42, p. 362 .
}

perturbation factors $G_{k}(t)$ are given by

$$
G_{k}(l)=\frac{1}{2 k+1} \sum_{\mu=-k}^{k} G_{k k^{\mu \mu}}(t)
$$

The following special cases exist:

(a) The static perturbation is zero and the fluctuating Hamiltonian is isotropic. The original density matrix may be chosen diagonal, with the result that the offdiagonal elements are always zero. The diagonal elements at time $t$ are given by Eq. (2.19). The perturbation factors for the powder source as well as for the single-crystal source are given by

$$
\begin{aligned}
& G_{k_{1} k_{2}}{ }^{0}(t)=\sum_{\alpha_{1} \beta_{1} r}(2 r+1)\left[\left(2 k_{1}+1\right)\left(2 k_{2}+1\right)\right]^{1 / 2} e^{-\lambda_{r} t} \\
& \quad \times\left(\begin{array}{rrr}
I & I & k_{1} \\
\alpha & -\alpha & 0
\end{array}\right)\left(\begin{array}{rrr}
I & I & r \\
\alpha & -\alpha & 0
\end{array}\right)\left(\begin{array}{rrr}
I & I & k_{2} \\
\beta & -\beta & 0
\end{array}\right)\left(\begin{array}{rrr}
I & I & r \\
\beta & -\beta & 0
\end{array}\right) .
\end{aligned}
$$

The sums over the vector coupling coefficients can be performed, yielding the final result for the angular correlation function (see Ref. 1)

$$
W^{\top}\left(\mathbf{k}_{\mathbf{1}}, \mathbf{k}_{\cdot 2}, t\right)=\sum_{k} A_{k}^{(1)} A_{k}^{(2)} e^{-\lambda_{k} t} P_{k}(\cos \theta)
$$

(b) The static perturbation is an axially symmetric field gradient, while the fluctuating perturbation is isotropic. The perturbation coefficients can be written for a single-crystal source as

$$
\begin{aligned}
& G_{k_{1} k_{2}}{ }^{\mu \mu}(t)=\sum_{\alpha}\left[\left(2 k_{1}+1\right)\left(2 k_{2}+1\right)\right]^{1 / 2} \\
& \quad \times\left(\begin{array}{rrr}
I & I & k_{1} \\
\alpha^{\prime} & -\alpha & \mu
\end{array}\right)\left(\begin{array}{rrr}
I & I & k_{2} \\
\alpha^{\prime} & -\alpha & \mu
\end{array}\right) e^{-(i / \hbar)\left(\alpha-\alpha^{\prime}\right) t} \\
& \quad \times\left[(2 r+1)\left(\begin{array}{rrr}
I & I & r \\
\alpha & -\alpha & 0
\end{array}\right)\left(\begin{array}{rrr}
I & I & r \\
\beta & -\beta & 0
\end{array}\right) e^{-\lambda_{r} t}+e^{-\lambda_{\alpha \alpha^{\prime} t}}\right],
\end{aligned}
$$

while for a crystalline powder source one obtains

$$
\begin{aligned}
G_{k}(t)=\frac{e^{-\lambda_{k} t}}{2 k+1}+\sum_{\alpha \neq \alpha^{\prime}}\left(\begin{array}{ccc}
I & I & k \\
\alpha^{\prime} & -\alpha & \mu
\end{array}\right)^{2} \\
\\
\quad \times e^{-\left[3 i\left(\alpha^{2}-\alpha^{\prime 2}\right) \omega\right.} Q^{t]} e^{-\lambda \alpha \alpha^{\prime} t},
\end{aligned}
$$

where $\omega_{Q}$ is the usual quadrupole frequency. ${ }^{1}$

(c) Finally, for the most general case treated here, that of an axially symmetric field gradient combined with a fluctuating anisotropic perturbation, we obtain 
in the single-crystal case the expression

$$
\begin{aligned}
& G_{k_{1} k_{2}}{ }^{\mu \mu^{\prime}}(t)=\sum_{\alpha} \sum_{r}\left(\begin{array}{rrr}
I & I & k_{1} \\
\alpha^{\prime} & -\alpha & \mu
\end{array}\right)\left(\begin{array}{rrr}
I & I & k_{2} \\
\alpha^{\prime} & -\alpha & \mu^{\prime}
\end{array}\right) \\
& \times b_{r \alpha \alpha^{\prime}} c_{r \beta \beta^{\prime}} e^{-\lambda_{r} t}+\sum_{\alpha}\left(\begin{array}{rrr}
I & I & k_{1} \\
\alpha^{\prime} & -\alpha & \mu
\end{array}\right)\left(\begin{array}{rrr}
I & I & k_{: 2} \\
\alpha^{\prime} & -\alpha & \mu^{\prime}
\end{array}\right) \\
& X e^{-i\left(\alpha-\alpha^{\prime}\right) t} e^{-\lambda \alpha \alpha^{\prime} t},
\end{aligned}
$$

while in the crystalline-powder case the perturbation factor can be obtained from Eq. $\left(3.1^{\prime}\right)$.

It is interesting to note in the above formulas that the relaxation of that term in the angular correlation which does not oscillate in time is always connected with the diagonal elements of the density matrix, while the relaxation of the terms which oscillate in time is related only to the off-diagonal elements of the density matrix. The same separation between the two different types is observed in NMR experiments, where the behavior of the diagonal elements determines the relaxation times $T_{1}$ while the off-diagonal elements determine the relaxation times $T_{2}$.

It is, furthermore, interesting to note that if it is possible to measure the relaxation constants of all of the different oscillating parts of the perturbation factor, a fairly direct relationship between different elements of the relaxation matrix $R$ can be determined, and this can provide important qualitative information as to the structure of the relaxation mechanism. On the other hand, the structure of the nonoscillating part of the perturbation factor, especially whether it consists of more than one exponential, can provide information on the symmetry of the fluctuating perturbation.

We note that the equations in this paper have been derived for the particular case of an odd- $A$ nucleus in an axially symmetric static field gradient. We have further emphasized quadrupole-type fluctuating fields, although the formulas have been derived for a more general form. The generalization to more complex situations such as asymmetric field gradients or even- $A$ nuclei can be done in a manner similar to that used for the case of static perturbations (Ref. 1). In making this generalization, it would be necessary to check the sym- metries of the relaxation matrix and to watch for degeneracies or for equivalence of energy separations in each special case.

\section{CONCLUSION}

We have discussed the application of the BlochWagnness-Redfield theory of nuclear relaxation to the interpretation of angular correlation measurements in solid sources in the presence of fluctuating perturbations It has been shown that the theory permits derivation of the form of the perturbation factor, and allows one to predict the structure and the number of the different relaxation coefficients involved for various special cases. In particular, it also allows one to use the extensive literature of NMR in interpreting, in a very simple way, angular correlation experiments involving relaxation.

A quite extensive treatment of the effect of relaxation on the linewidth of Mössbauer spectra has been made by Blume and Tjon ${ }^{12}$ from a slightly different point of view, and these authors show that their results can be directly applied to the calculation of the perturbation coefficients in time dependent angular correlations. The technique differs from that adopted here in that it is basically model dependent. It has, however, the advantage that it can be used to discuss situations in which $t$ is comparable to or shorter than the correlation time $\tau_{c}$, and thus allows the study of several cases of experimental interest that can not be treated by the formulas presented here.

The formalism developed in this paper has been used to discuss measurements of the time-dependent angular correlation of radiations from $\mathrm{Hf}^{181}$ included in the compound $\mathrm{HfF}_{7}\left(\mathrm{NH}_{4}\right)_{3}$ providing useful information about the molecular structure of this compound. ${ }^{13}$

\section{ACKNOWLEDGMENTS}

One of the authors (P.daR.A.) thanks the Conselho Nacional de Pesquisas (Brasil) for a research fellowship and another one (J.D.R.) is indebted to the Pan American Union for support during this work.

${ }_{12}$ M. Blume and J. A. Tjon, Phys. Rev. 165, 446 (1968).

${ }^{13} \mathrm{P}$. da R. Andrade, Thesis for the degree of "Doutor em Ciências," Faculty of Sciences, Porto Alegre, 1968 (unpublished). 